\title{
Pediatric Portal Interventions
}

\author{
Rajiv N. Srinivasa, MD ${ }^{1}$ Jeffrey Forris Beecham Chick, MD, MPH, DABR ${ }^{1}$ Noah Chen, BS ${ }^{2}$ \\ Joseph J. Gemmete, MD, FSIR ${ }^{1}$ Wael A. Saad, MD, FSIR ${ }^{1} \quad$ Narasimham L. Dasika, MD ${ }^{1}$ \\ Ravi N. Srinivasa, $\mathrm{MD}^{1}$
}

1 Division of Vascular and Interventional Radiology, Department of Radiology, University of Michigan Health System, Ann Arbor, Michigan

2 Department of Biological Chemistry, School of Medicine, University of Michigan, Ann Arbor, Michigan

Address for correspondence Ravi N. Srinivasa, MD, Department of Radiology, Division of Vascular and Interventional Radiology, University of Michigan Health System, 1500 East Medical Center Drive, Ann Arbor, Ml 48109 (e-mail: medravi@gmail.com).

Semin Intervent Radiol 2018;35:143-150

${ }^{*}$ CME credit is not offered for this article

Pediatric portal interventions pose different challenges when compared with those in adults. Furthermore, the workup and clinical management of children in the preoperative, operative, and postoperative periods are different from that of the adult population with liver disease requiring a portal intervention. Decision-making processes should be executed differently in children based on these factors. Often, pediatric patients requiring portal interventions are transferred to larger academic institutions with standalone children's hospitals and child-specific liver transplant initiatives. Thus, smaller institutions may not have the personnel or systems in place to properly perform these interventions. As an alternative to surgical splenorenal or mesocaval shunts, transjugular intrahepatic portosystemic shunts (TIPS) may be performed in children to manage variceal bleeding and ascites. Other portal interventions including portal vein recanalization, variceal sclerotherapy, splenic embolization, and congenital portosystemic shunt embolization may be performed as well. ${ }^{1}$

\section{Etiological Differences}

Children exhibit symptoms with similar manifestations of hepatic disease to adults such as variceal bleeding, ascites, and hepatic hydrothorax. The etiology of portal hypertension in children, however, is unique compared with adults. In adults, alcohol consumption, nonalcoholic steatohepatitis, and viral hepatitis leading to cirrhosis are the most common causes of portal hypertension. In children, on the other hand, the etiology of portal hypertension is often congenital or acquired in origin. After leukemia treatment, for example, children may present with post-chemotherapy-related liver disease. Additionally, in the setting of chronic liver disease, long-term percutaneous enteral nutrition may result in peristomal variceal formation which are prone to bleeding. Children with biliary atresia undergoing liver transplant may experience transplant-related complications such as hepatic artery occlusion or portal vein anastomotic narrowing. Dehydration in infants and small children is also a potential etiology in sinistral portal hypertension resulting from splenic thrombosis. Children may also have congenital portosystemic communications (Abernethy malformations and other variants) which result in hepatopulmonary syndrome. All of these conditions may be managed by minimally invasive interventional approaches such as TIPS, portal vein recanalization, variceal sclerotherapy, splenic embolization, and congenital portosystemic shunt embolization mitigating the need for open surgical operations.

\section{Transjugular Intrahepatic Portosystemic Shunts}

The techniques for placement of TIPS are similar in both adults and children. However, the anatomy and physiology of children is different from that of adults and this must be taken into consideration when placing a TIPS. The hepatic and portal veins are smaller in a child, making cannulation and access into the portal vein more difficult. Furthermore, many TIPS kits are designed for adults and given the smaller size of children, this is not always optimal for the performance of the procedure. In addition, fluid volume status, blood loss, contrast dose, cardiac status, airway management, anesthesia, and radiation dose all must be taken more into consideration when placing a TIPS in a small child. Since children with portal hypertension have a longer life expectancy than adults, TIPS placement will usually serve as a bridge to liver transplantation. ${ }^{1,2}$ After placement of the shunt, the child's growth must be taken into consideration, as the shunt may need to be revised as the child grows. Based on the severity of portal hypertension and patient-specific circumstances, transjugular, transhepatic, or percutaneous transhepatic sclerotherapy without placement of a TIPS may be performed to reduce the risk of hepatic encephalopathy or
Issue Theme Pulmonary Embolism; Guest Editors, Ronald Winokur, MD and David C. Madoff, MD
Copyright (c) 2018 by Thieme Medical Publishers, Inc., 333 Seventh Avenue, New York, NY 10001, USA. Tel: +1(212) 584-4662.
DOI https://doi.org/ 10.1055/s-0038-1642043. ISSN 0739-9529. 
worsening liver or right heart failure. ${ }^{1-3}$ These risks are particularly important to consider in children, as they are more vulnerable if proper screening and planning is not performed prior to TIPS placement.

In the setting of pediatric variceal hemorrhage, the first line of management is endoscopic injection of vasoconstrictor drugs, endoscopic banding or gluing of varices. A Sengstaken-Blakemore tube may also be required if hemorrhage is life-threatening. Medications to consider include vasopressin and other vasopressors, $\beta$-blockers, or octreotide along with supportive therapy including fluid resuscitation and blood transfusion.

The TIPS procedure in children is similar to that in adults. Internal jugular vein access is obtained under ultrasound and a 9- or 10-Fr, 40-cm sheath is placed, depending on the size of the child. A modified catheter, such as a MPA or C2 Cobra, with the tip cut off may be used to select either the right or middle hepatic vein. A hepatic venogram and balloon occlusion carbon dioxide venogram are performed to map the hepatic and portal venous systems. A Colapinto needle or Rosch-Uchida set is used to puncture a portal venous branch from the hepatic vein. Needle entry is confirmed by injection of contrast after which a wire is placed into the portal venous system. A catheter is then passed into the portal venous system followed by a stiff wire.

In smaller prepubertal children who have not yet obtained their maximum growth potential, an uncovered balloonexpandable stent (Express; Boston Scientific; Marlborough, MA), or a balloon-expandable covered stent (iCast; Atrium Medical; Merrimack, NH), is advantageous in providing adequate, but not excessive, portal decompression while at the same time allowing for subsequent expansion of the shunt to an 8- to 10-mm diameter as the child grows (-Fig. 1). Typically, an 8- to 10 -mm diameter TIPS, usually a Viatorr stentgraft, may be placed in an adolescent child into adulthood.

Variceal sclerotherapy using alcohol, sodium tetradecyl sulfate, or coils should also be performed concomitantly with TIPS placement in the setting of variceal bleeding. Postoperatively, patients should be assessed for complications, which mirror those in adults, including hepatic encephalopathy, bleeding, and liver failure.

Implementing TIPS procedures in pediatric transplant recipients may be especially difficult because they often have split grafts. In particular, left hepatic lobe split grafts pose a definitive challenge because of the size of the liver and vessels as well as the different orientation of the vessels, as most operators are accustomed to right-sided TIPS placement. Split left lobe grafts, particularly segment $2 / 3$ grafts, rotate, grow, and may have vessels with unconventional orientations as well. ${ }^{4-6}$

\section{Portal Vein Recanalization}

The most common reason for liver transplantation in young children is biliary atresia. The rate of postoperative portal vein thrombosis after pediatric liver transplantation is as high as $16 \%{ }^{7}$ Patients presenting 30 days after transplant with portal vein thrombosis will frequently have variceal



Fig. 1 5-year-old patient status post liver transplant for biliary atresia complicated by portal hypertension and varices. One week following the initial transhepatic embolization procedure the patient had recurrent hematemesis and anemia and there was concern for recanalization of varices. (a) Right hepatic vein access was obtained using a selective catheter and a 10 French braided vascular sheath (Cook Medical). A Colapinto needle was used to puncture the right portal vein (white arrow). Embolization material within the esophageal varices is again seen (white arrowheads). (b) A wire was advanced into the portal venous system into the superior mesenteric vein (white arrow). (c) Digital subtraction venography of the portal venous system through a marker flush catheter demonstrates persistent filling of the esophageal varices through a collateral network from the superior mesenteric vein (white arrows). (d) An $8 \mathrm{~mm} \times 37 \mathrm{~mm}$ balloon-expandable Express (Boston Scientific; Marlborough, MA) stent (white arrow) was deployed between the right hepatic and right portal vein for creation of a transjugular intrahepatic portosystemic shunt. The portosystemic gradient dropped from $24 \mathrm{mmHg}$ to $10 \mathrm{mmHg}$ following creation of the TIPS, and there was decompression of the collateral mesenteric vessels supplying the patient's varices.

bleeding. Pediatric portal vein recanalization is challenging due to the small caliber vessels, altered surgical anatomy, and postoperative fibrosis. Similar methods used in adults, however, may be used in children to perform recanalization of an occluded portal vein.

Both transhepatic and transsplenic ${ }^{1,8}$ approaches may be used to achieve portal vein recanalization. Transhepatic or transsplenic access is obtained and a 4-Fr sheath is placed. Venography is performed to determine the degree of stenosis or occlusion. The occlusion or stenosis can be crossed using a variety of wires and catheters. If blunt recanalization techniques fail, sharp recanalization can be considered with the back end of a wire or long 22-gauge Chiba needle. Once the occlusion is crossed, manometry and balloon angioplasty are performed. Depending on the flow in the portal and splenic vein, stent placement may be necessary. Furthermore, some patients may require TIPS to improve flow in the portal vein.

Balloon-expandable stents are preferred in the portal vein to allow for further expansion as the child ages (-Fig. 2). 


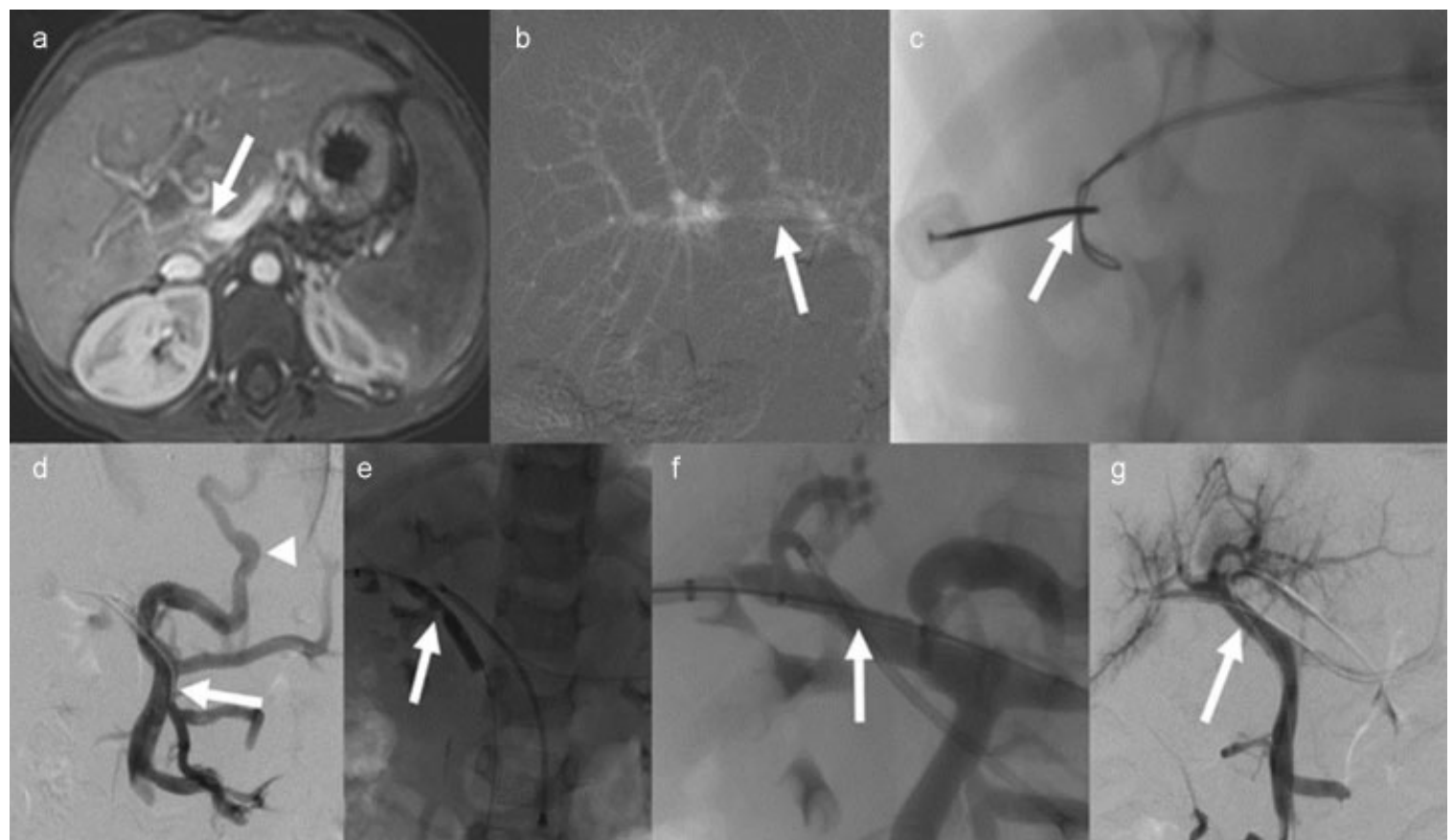

Fig. 2 A 3-year-old patient status post liver transplant for neonatal cholestasis secondary to bile salt export pump (BSEP) deficiency who developed delayed portal vein thrombosis 3 years after transplantation. The patient had worsening splenomegaly and paraesophageal varices. (a) Axial contrast-enhanced magnetic resonance image of the abdomen demonstrated subacute occlusion of the portal vein (white arrow). (b) Following access into the left portal vein (white arrow), carbon dioxide digital subtraction angiography confirmed occlusion of the main portal vein. Multiple attempts to traverse the occlusion from a left portal vein approach were unsuccessful due to unfavorable angles. (c) A snare was placed into the right portal vein from the left sided access and a 21 gauge Chiba needle (Cook Medical) was used to obtain percutaneous access through the snare (white arrow). (d) An angled catheter and Glidewire (Terumo Medical) were used to traverse the obstruction and obtain access into the superior mesenteric vein (white arrow). Digital subtraction angiography confirms occlusion of the main portal vein with filling of paraesophageal varices (white arrowhead). (e) Balloon angioplasty of the main portal vein was performed with a $6 \mathrm{~mm} \times 4 \mathrm{~cm}$ Symmetry (Boston Scientific) balloon (white arrow) followed by an $8 \mathrm{~mm}$ Mustang (Boston Scientific) balloon (not pictured). (f) A $7 \mathrm{~mm} \times 22 \mathrm{~mm}$ Atrium iCast (Atrium Medical; Merrimack, NH) stent was then positioned at the level of the main portal vein occlusion (white arrow) and deployed. It was then balloon dilated to $10 \mathrm{~mm}$. (g) Digital subtraction portal venography following stent deployment reveals a widely patent portal vein (white arrow) with decompression of the paraesophageal varices. The gradient between the superior mesenteric vein and the intrahepatic portal veins was initially $10 \mathrm{mmHg}$ and dropped to zero following stent placement.

Similarly, if a TIPS is placed, the tract is lined with a balloonexpandable covered stent to allow for expansion as the child grows into adolescence and adulthood.

The length, degree of occlusion, and chronicity may pose significant obstacles that may make recanalization challenging, possibly necessitating multiple accesses or sharp recanalization. For these conditions, periodic ultrasound or magnetic resonance imaging follow-up is recommended to ensure no recurrent stenosis or thrombosis. Early detection of stenosis and thrombosis is important as treatment becomes more difficult with increasing chronicity. Angioplasty with thrombolysis may be able to treat pure acute or subacute thrombosis, but stenting may be required in the setting of chronic occlusions.

Embolization of the transhepatic or transsplenic tract should be achieved with Gelfoam pledgets, coils, or $n$-butyl cyanoacrylate glue. ${ }^{9}$ Bleeding postprocedure is usually from the transhepatic or transsplenic access and patients should be admitted postoperatively and monitored for signs of bleeding. Another potential cause of bleeding is an abrupt increase in intra-abdominal pressure after the patient awakens from general anesthesia, typical in pediatric cases. One method for reducing this risk is to hold pressure at the puncture site while the patient is awakening from anesthesia, particularly when a transsplenic approach is used. A follow-up ultrasound is recommended the day following the procedure to detect any acute complications developing from the access and to confirm stent patency.

\section{Variceal Sclerotherapy}

Portal hypertension in the pediatric patient may lead to esophageal varices, enteric varices, gastric varices, and spontaneous splenorenal shunts. Enteric varices are relatively uncommon, occurring in only $5 \%$ of pediatric patients with portal hypertension. ${ }^{2}$ Surgery, TIPS placement, percutaneous embolization, and endoscopic sclerotherapy are all options for the treatment of enteric varices. In patients with a portal vein stenosis, stent placement may be helpful to decompress the varices, prevent rebleeding, and normalize flow to the liver. ${ }^{1}$

Given that the risk of radiation-induced injury for pediatric patients is higher, magnetic resonance imaging with magnetic resonance venography of the portal venous system 
should be obtained preoperatively to define the anatomy and provide a roadmap for intervention to minimize the radiation dose from the procedure. Although treatment of enteric varices is similar to adults, in small children, TIPS placement should be avoided, if possible, given the growth potential of the child.

In pediatric patients, transhepatic access into the portal venous system is obtained under ultrasound with a 21- or 22-gauge needle (-Fig. $\mathbf{3}$ ). Through this access, a balloon is negotiated into the vein filling the varices. The balloon is inflated and transvenous antegrade embolization of the varices is performed with foamed sodium tetradecyl sulfate followed by coil placement (-Fig. 4). If the varices are superficial, however, $1 \%$ STS may be more appropriate than $3 \%$ STS to prevent possible necrosis of the overlying skin. After variceal sclerotherapy, the transhepatic portal vein tract is embolized with Gelfoam pledgets, coils, or $n$-butyl cyanoacrylate glue.

In the setting of gastroesophageal varices or isolated gastric varices, balloon-occluded retrograde transvenous obliteration has been described in children through a gastrorenal shunt. ${ }^{3,10}$ Conventional balloon-occluded retrograde transvenous obliteration in pediatric patients with gastric variceal bleeding is technically feasible similar to conventional balloon-occluded retrograde transvenous obliteration in adults, though with smaller balloon sizes and less foam sclerosant volumes. ${ }^{3}$ In the setting of partial or complete portal vein thrombosis or complete splenic vein thrombosis, partial splenic artery embolization may also be performed as an adjunct to variceal sclerosis. ${ }^{3}$

\section{Splenic Artery Embolization}

Partial splenic artery embolization is typically used for the treatment of hypersplenism or symptomatic portal hypertension in the setting of thrombocytopenia. Furthermore, partial splenic artery embolization may be used to augment other treatment options for portal hypertension (e.g., conventional balloon-occluded retrograde transvenous obliteration) or serve as a less invasive treatment option for managing variceal bleeding without placing a shunt. Recent recanalization of the portal vein or splenic vein is a relative contraindication to splenic artery embolization, as the procedure results in reduced inflow to the splenic and portal veins and therefore may predispose to repeat thrombosis.

The celiac artery is selected and arteriography performed to assess the anatomy, tortuosity of the splenic artery, and patency of the splenic and portal veins. Based on the results, partial splenic embolization should be performed with particles (-Fig. 5). Ampicillin and gentamicin may be

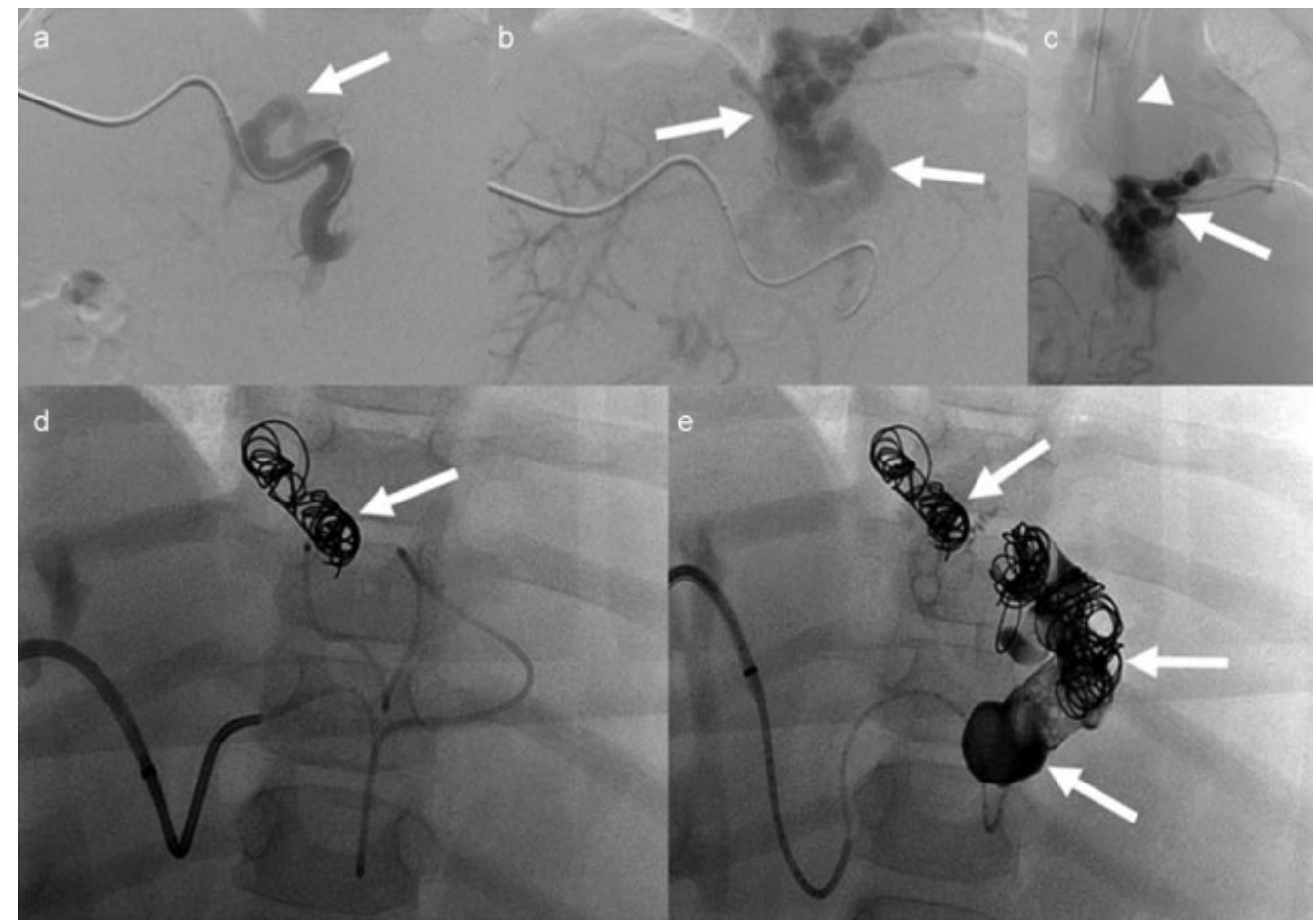

Fig. 3 5-year-old patient status post liver transplant for biliary atresia complicated by portal hypertension and varices. Patient had multiple prior banding procedures for esophageal varices. (a, b) Percutaneous transhepatic access into the portal system was obtained with placement of a 7 French, $10 \mathrm{~cm}$ Pinnacle sheath (Terumo Medical; Tokyo, Japan). Upon contrast injection there is filling of a coronary vein varix seen on early (white arrow, a) and delayed images (white arrows, b). (c) Selective cannulation of the coronary vein varix was performed with a 5 French SOS Omni Select catheter (Angiodynamics; Latham, NY) with contrast injection revealing robust filling of esophageal varices (white arrow) with drainage into the azygous system (white arrowhead). (d) A Renegade high flow microcatheter was advanced further into the varix and coil embolization performed with multiple 8 and $10 \mathrm{~mm}$ (white arrow) MicroNester coils (Cook Medical; Bloomington, IN) to slow the drainage into the systemic circulation prior to sclerotherapy. (e) $4 \mathrm{~mL}$ of a mixture of foamed $3 \%$ sodium tetradecyl sulfate (Mylan; Canonsburg, PA) was then injected into the varices, followed by additional coils (white arrows) and n-butyl cyanoacrylate (TRUFILL; Codman Neuro; Raynham, MA). 


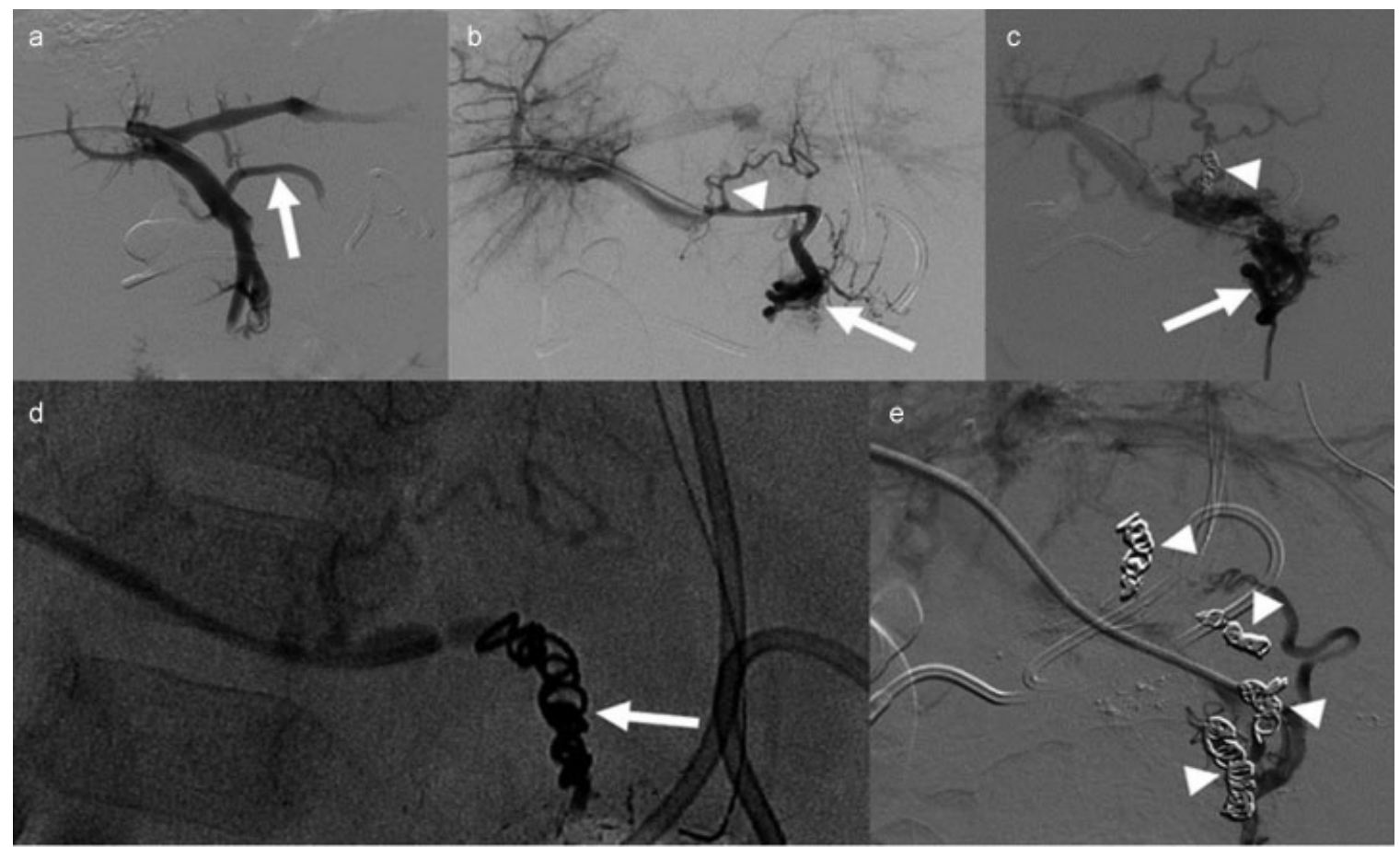

Fig. 4 A 20-month-old patient with autosomal recessive polycystic kidney disease status post bilateral nephrectomies now with liver fibrosis and portal hypertension. Patient had bleeding gastric stomal varices. (a) Transhepatic access was obtained into the portal venous system with digital subtraction venography which demonstrated filling of a left gastric vein (white arrow). (b, c) Digital subtraction venography of the portal vein demonstrating a varix (b, white arrowhead) which was subsequently coil embolized (c, white arrowhead). Images also demonstrated filling of the gastric stomal varices through collaterals from the left gastric vein (b and c, white arrows). (d) Sclerotherapy of the varices was performed with $0.8 \mathrm{~mL}$ of dehydrated alcohol with subsequent coil embolization of the left gastric vein (white arrow). (e) Multiple additional splenic collaterals were also coil embolized (white arrowheads).

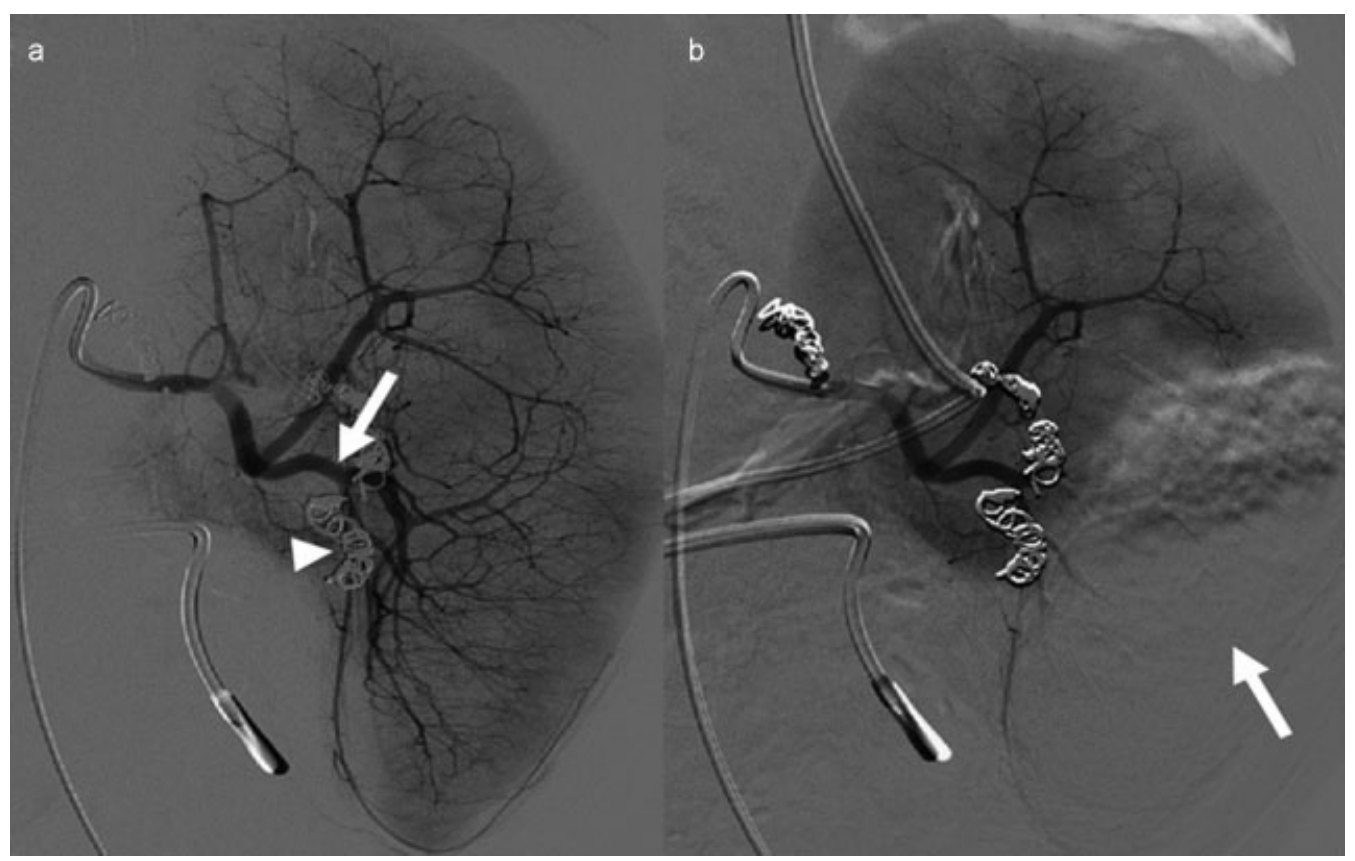

Fig. 5 A 20-month-old patient with autosomal recessive polycystic kidney disease status post bilateral nephrectomies now with liver fibrosis and portal hypertension. Five days after initial embolization, there was persistent oozing from varices and partial splenic embolization was recommended. (a) Splenic arteriography through a $5 \mathrm{Fr}$ Shepherd Hook (Angiodynamics) catheter demonstrated robust filling of the spleen with splenomegaly. The lower pole splenic artery (white arrow) was noted to be a suitable target for partial embolization. Embolization coils from prior splenic vein collateral embolization are noted (white arrowhead). (b) Following embolization of the lower pole splenic artery with 100-300 micron particles (Embospheres; Merit Medical; South Jordan, UT), there is appropriate devascularization of the lower pole of the spleen (white arrow). 
administered intra-arterially to reduce the risk of splenic abscess development. The lower pole of the spleen should be embolized to prevent irritation of the diaphragm and the possible development of a pleural effusion, poor inspiratory effort leading to pneumonia or atelectasis, or intractable hiccups.

Postembolization syndrome, flank pain, and liquefactive infarction with infection and abscess formation are the most common complications seen after splenic artery embolization. Unlike in adults, flank pain after pediatric splenic artery embolization may be a source of considerable morbidity and may require hospital admission for hydration and pain control. To reduce the risk of postprocedure pain and liquefactive infarction, multiple embolization sessions should be performed with 25 to $35 \%$ of the splenic parenchymal infarcted per session. The interval between each session should be at least 6 to 8 weeks. If embolization of a large portion of the spleen is performed, then the platelet count should be monitored for thrombocytosis. A high platelet count may lead to thrombogenic states and thrombose the portal and splenic veins.

\section{Congenital Portosystemic Shunt Embolization}

Congenital intrahepatic portosystemic shunts may lead to significant flow dynamic alteration in the portal circulation, resulting in elevated right heart pressures and potentially high-output cardiac failure. ${ }^{11-21}$ Congenital intrahepatic portosystemic shunts represent an abnormal intrahepatic connection between the hepatic veins and the portal vein. These represent persistence of an embryologic communication between the vitelline veins of the omphalomesenteric system and the sinus venous due to a focal absence of sinusoid formation.
Congenital extrahepatic portosystemic shunts, such as Abernethy malformations, represent another type of congenital portosystemic shunt. ${ }^{11-21}$ Abernethy malformations are typically classified into two main categories. Type I involves complete diversion of portal blood flow away from the liver resulting in no portal blood flow to the liver. Type II involves partial diversion of portal flow away from the liver with some residual inline portal flow into the liver. The further subclassifications of Type II Abernethy malformations include Type IIa, where the fistula arises from the portal vein branches (either left, right, or through a patent ductus venosus); Type IIb, where the fistula arises from the main portal vein, including its bifurcation or the confluence of its tributaries; and Type IIc, where the fistula arises from the tributaries of the portal vein (splenic or mesenteric veins). ${ }^{1,5,6,10}$

Patients with hepatopulmonary shunts may develop hepatopulmonary syndrome from efflux of venous effluents from the liver into the lungs. ${ }^{22}$ The mechanism is not fully elucidated; however, it likely involves both upregulation and production of nitric oxide and the inability of the liver to metabolize vasoactive mediators, resulting in ventilation and perfusion mismatches and shunting through pulmonary arteriovenous malformations. These patients commonly present with cyanosis, polycythemia, clubbing, and limited exercise tolerance from hypoxemia. Cerebral abscesses, systemic embolization, and pulmonary hemorrhage are more serious potential complications. ${ }^{9,10}$

Embolization of congenital intrahepatic portosystemic shunts is frequently indicated due to clinical symptoms, including hepatic encephalopathy secondary to hyperammonemia and hepatopulmonary syndrome. If complete single-stage embolization is not feasible due to poorly developed portal or hepatic veins, then staged embolization or liver transplantation should be considered. Hepatic and

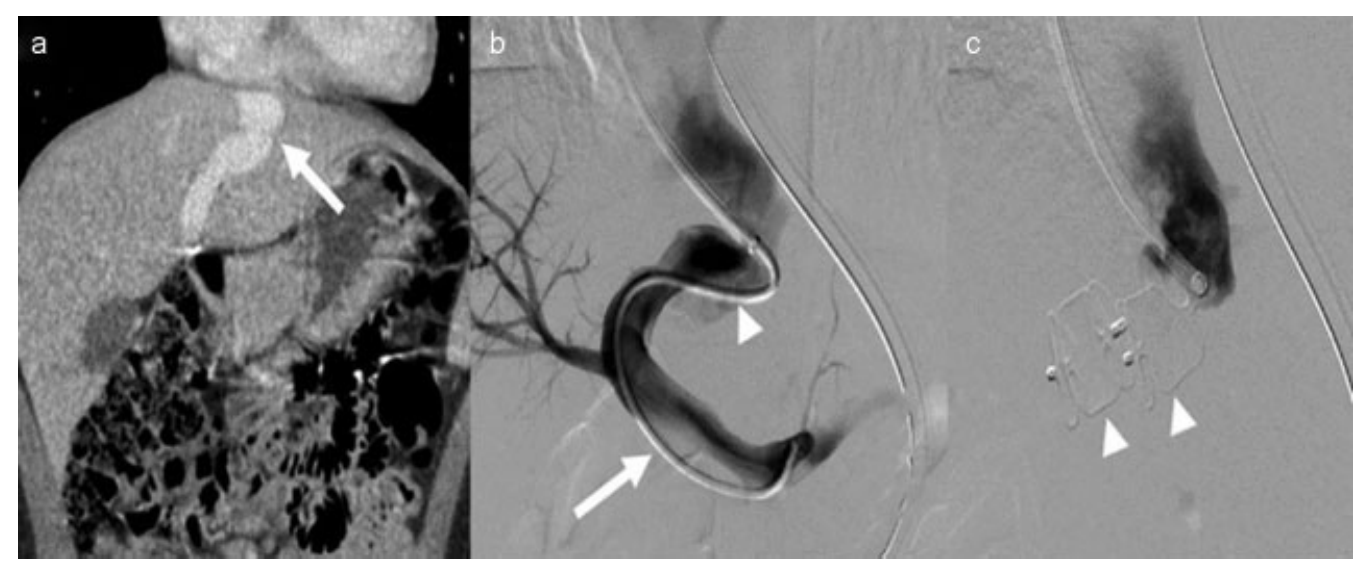

Fig. 6 A 7-year-old patient with a 1-year history of shortness of breath with concern for hepatopulmonary syndrome. The patient had a baseline oxygen saturation of $82-83 \%$. (a) Coronal computed tomography image of the abdomen demonstrated an abnormal portohepatic communication (white arrow) consistent with a congenital portosystemic shunt. (b) Digital subtraction venography after cannulation of the portosystemic shunt demonstrating robust filling of a communication between the portal vein (white arrow) and hepatic vein (white arrowhead). The intrahepatic portal veins were assessed and deemed to be well-developed and of adequate size to allow for single stage closure of the shunt. (c) Two second-generation Amplatzer (AVP2; St. Jude Medical; Saint Paul, MN) vascular plugs (white arrowheads) were used to close the shunt with final venography showing no further flow through the shunt. The patient's oxygenation progressively improved following embolization. 
portal venography and manometry should be performed before and after a trial balloon occlusion of the shunt prior to definitive embolization. The Amplatzer Vascular Plug (AVP2) is the easiest method for occlusion of these large shunts (-Fig. 6); however, coils may also be used. Early treatment is critical because embolization becomes more difficult as the shunt enlarges.

\section{Conclusion}

Portal interventions in adults are common and well described, but pediatric portal interventions are less commonly detailed. TIPS, portal vein recanalization, variceal sclerotherapy, splenic embolization, and congenital portosystemic shunt embolization may be performed in children similar to adults; however, careful consideration is needed to accommodate for physiological differences and the growth potential of the child.

\section{References}

1 Srinivasa RN, Dasika NL, Jo A, Saad WEA. Endovascular management of portal hypertension complications in the pediatric population. In: Saad WEA, ed. Portal Hypertension: Imaging, Diagnosis, and Endovascular Management. 3rd ed. New York, NY: Thieme; 2017:225-232

2 Heyman MB, LaBerge JM, Somberg KA, et al. Transjugular intrahepatic portosystemic shunts (TIPS) in children. J Pediatr 1997; 131(06):914-919

3 Saad WE, Anderson CL, Patel RS, et al. Management of gastric varices in the pediatric population with balloon-occluded retrograde transvenous obliteration (BRTO) utilizing sodium tetradecyl sulfate foam sclerosis with or without partial splenic artery embolization. Cardiovasc Intervent Radiol 2015;38(01):236-241

4 Saad WE, Davies MG, Lee DE, et al. Transjugular intrahepatic portosystemic shunt in a living donor left lateral segment liver transplant recipient: technical considerations. J Vasc Interv Radiol 2005;16(06):873-877

5 Saad WE, Darwish WM, Davies MG, Waldman DL. Transjugular intrahepatic portosystemic shunts in liver transplant recipients for management of refractory ascites: clinical outcome. J Vasc Interv Radiol 2010;21(02):218-223

6 Saad WE, Darwish WM, Davies MG, et al. Transjugular intrahepatic portosystemic shunts in liver transplant recipients: technical analysis and clinical outcome. AJR Am J Roentgenol 2013;200 (01):210-218

7 Waits SA, Wojcik BM, Cai S, Mathur AK, Englesbe MJ. Portal vein thrombosis and outcomes for pediatric liver transplant candi- dates and recipients in the United States. Liver Transpl 2011;17 (09):1066-1072

8 Chick JFB, Jo A, Dasika N, Saad WE, Srinivasa RN. Transsplenic endovascular recanalization and stenting of a completely occluded portal vein with jejunal variceal embolization in a pediatric liver transplant recipient. Pediatr Radiol 2017;47(08):1012-1015

9 Saad WE, Madoff DC. Percutaneous portal vein access and transhepatic tract hemostasis. Semin Intervent Radiol 2012;29(02): 71-80

10 Saad WE, Kitanosono T, Koizumi J, Hirota S. The conventional balloon-occluded retrograde transvenous obliteration procedure: indications, contraindications, and technical applications. Tech Vasc Interv Radiol 2013;16(02):101-151

11 Alonso J, Sierre S, Lipsich J, Questa H, Faella H, Moguillansky S. Endovascular treatment of congenital portal vein fistulas with the Amplatzer occlusion device. J Vasc Interv Radiol 2004;15(09): 989-993

12 Florio F, Nardella M, Balzano S, Giacobbe A, Perri F. Congenital intrahepatic portosystemic shunt. Cardiovasc Intervent Radiol 1998;21(05):421-424

13 Gallego C, Miralles M, Marín C, Muyor P, González G, GarcíaHidalgo E. Congenital hepatic shunts. Radiographics 2004;24(03): 755-772

14 Konstas AA, Digumarthy SR, Avery LL, et al. Congenital portosystemic shunts: imaging findings and clinical presentations in 11 patients. Eur J Radiol 2011;80(02):175-181

15 Lautz TB, Tantemsapya N, Rowell E, Superina RA. Management and classification of type II congenital portosystemic shunts. J Pediatr Surg 2011;46(02):308-314

16 Lee SA, Lee YS, Lee KS, Jeon GS. Congenital intrahepatic portosystemic venous shunt and liver mass in a child patient: successful endovascular treatment with an Amplatzer vascular plug (AVP). Korean J Radiol 2010;11(05):583-586

17 Morikawa N, Honna T, Kuroda T, et al. Resolution of hepatopulmonary syndrome after ligation of a portosystemic shunt in a pediatric patient with an Abernethy malformation. J Pediatr Surg 2008;43(02):e35-e38

18 Park JH, Cha SH, Han JK, Han MC. Intrahepatic portosystemic venous shunt. AJR Am J Roentgenol 1990;155(03):527-528

19 Scalabre A, Gorincour G, Hery G, Gamerre M, Guys JM, de Lagausie P. Evolution of congenital malformations of the umbilical-portalhepatic venous system. J Pediatr Surg 2012;47(08):1490-1495

20 Stringer MD. The clinical anatomy of congenital portosystemic venous shunts. Clin Anat 2008;21(02):147-157

21 Nacif LS, Paranaguá-Vezozzo DC, Galvão FHF, et al. Significance of CT scan and color Doppler duplex ultrasound in the assessment of Abernethy malformation. BMC Med Imaging 2015;15:37

22 Chick JFB, Reddy SN, Yu AC, et al. Three-dimensional printing facilitates successful endovascular closure of a Type II Abernethy malformation using an Amplatzer atrial septal occluder device. Ann Vasc Surg 2017;43:311.e15-311.e23 